\title{
Impact of Post Mastectomy Radiotherapy on the Silicone Breast Implant
}

Louise J Magill ${ }^{1}$, Kate Ricketts ${ }^{1}$, Mohammed Keshtgar ${ }^{1,2}$, Ash Mosahebi ${ }^{1,2}$, Gavin Jell $^{1}$

1: Division of Surgery and Interventional Science, Royal Free Campus, University

College London, Pond Street, London NW3 2QG.

2: Department of Plastic and Reconstructive Surgery, Royal Free London NHS

Foundation Trust, Pond Street, London, NW3 2QG

Key words: Post Mastectomy Radiation Therapy, Breast Implant, Silicone Implant, Breast Reconstruction, Failure

Financial Support: nil

Words:

\section{Corresponding author:}

Louise J Magill,

Division of Surgery and Interventional Science, Royal Free Campus, University

College London,

$9^{\text {th }}$ Floor Royal Free Hospital,

Pond Street,

London,

NW3 2QG.

Email: louise.magill.14@ucl.ac.uk 


\begin{abstract}
Implant based reconstruction accounts for over half of breast reconstruction performed in the UK. Patients with implant based breast reconstructions undergoing post mastectomy radiotherapy are at increased risk of capsular contracture and reconstructive failure. This study sought to determine the effect of treatment dose radiotherapy on the bulk mechanical, surface chemical properties of silicone implants as well as their cellular response. Silicone breast implant shells were submitted to treatment dose radiotherapy, 2.67Gy (one daily fraction) and 40.05Gy (15 fractions) using non-irradiated shells as controls. Bulk mechanical and surface chemical properties of the shells were evaluated using tensile and tear testing, attenuated total reflectance - fourier transform infrared spectroscopy (ATR-FTIR), water contact angle measurements. HDFa cells were seeded on the shells and Alamar Blue assay was performed to study cell metabolic activity. Cell morphology was evaluated using phalloidin and DAPI staining. There was no significant difference in tensile, tear jstrength and Young's modulus however there was reduction in maximum elongation following irradiation. Irradiation of the shells did not significant alter spectroscopy measurements nor wettability of the shells. Cell metabolism was not significantly affected by irradiation. Further analysis is warranted of the micromechanical properties to fully elucidate the effect of irradiation on the breast implant which could explain the increased rate of capsular contracture and reconstructive failure in patients undergoing post-mastectomy radiotherapy.
\end{abstract}




\section{Introduction}

Rates of mastectomy and breast reconstruction are increasing, even in patients who are deemed suitable for breast conserving surgery and those with early stage disease $[1,2]$. Breast reconstruction offers improved psychological, body-image, emotional and sexual well-being for patients undergoing mastectomy [3] and therefore is offered to those patients deemed suitable. Over half of breast reconstructions performed in the UK are implant based. There is increasing evidence of the benefits of post mastectomy radiotherapy (PMRT) in reducing loco-regional recurrence in certain cohorts of patients $[4,5]$. The recent guidelines published by the American Society of Surgical Oncology advise all patients with T1-2 tumours and 1-3 positive lymph nodes should be considered for PMRT as well as patients with T1-2 tumours with one positive node on sentinel node biopsy who do no undergo further axillary clearance should be considered [6].

However, IBBR is associated with long term complications including most commonly capsular contracture, causing breast distortion, pain and firmness as well as implant rupture and gel leakage. Capsular contracture has been reported to occur in $24.6 \%$ of patients at ten years in patients undergoing implant based reconstruction [7]. Silicone implants have also been associated with the development of autoimmune/inflammatory syndrome induced by adjuvants (ASIA) characterized by a deficient humoral response leading to symptoms of fatigue, myalgia, arthralgia, cognitive impairment and neurological manifestations [8]. TThe exact mechanism of the cause of breast implant failure is unknown and several factors have been postulated in both augmentation and reconstructive procedures including surgical handling of implant, biofilm formation, peri-operative haematoma, exposure to silicone and peri-operative radiotherapy $[9,10]$ Post mastectomy radiotherapy delivered to the permanent implant increases the rate of capsular contracture, implant failure and revisional surgery [11-13]. Moreover, it is associated with poor cosmetic outcome and patient satisfaction [13-15].

The influence of radiation therapy on the material chemical properties is unclear, with previous studies showing that radiation had no significant effect upon the surface and bulk properties of PDMS based materials [16,17], but others demonstrating change in the surface chemical properties following treatment dose radiation (50 Gy in 25 
fractions) of commercially available silicone breast implants [18]. The aim of this study was to examine the effect of treatment dose radiation therapy upon the mechanical and surface chemical properties as well as the cellular response to silicone breast implants following irradiation to gain a deeper understanding of the role of radiation therapy in the development of capsular contracture and breast implant failure. 


\section{Methods}

\section{Preparation and Irradiation of Silicone Breast Implant Shells}

Un-implanted textured silicone breast implants (Mentor Siltex ${ }^{\mathrm{TM}}$ Contour Profile ${ }^{\mathrm{TM}}$ Becker TM 35 Expander, Cohesive II ${ }^{\mathrm{TM}}$, Lot 6811381) were used. The implant inner gel was removed and the outer shells were subjected to radiation. The radiation delivered to the implants was based upon the recommended chest wall dosing schedule for patients with invasive breast cancer after mastectomy (40.05Gy in 15 fractions) (Recht 2001..]. The breast implant shells were then categorized into three groups according to full treatment dose radiotherapy (40.05 Gy), one daily fraction dose radiotherapy (2.67 Gy) and a non-irradiated shell was used as the control.

The implant shells were surrounded by blocks and adjuncts to simulate surrounding soft tissue and radiated at a rate of $6 \mathrm{~Gy} / \mathrm{min}$ courtesy of the Department of Radiotherapy, Royal Free Hospital, London.

\section{Mechanical Testing of Implant Shells}

All samples were measured using the Instron 5565 tensiometer equipped with a $500 \mathrm{~N}$ load (Instron, UK). From the implant shells, for each condition, six $20 \mathrm{~mm}$ x $4 \mathrm{~mm}$ dumbbell shaped specimens were for tensile testing and 3 crescent shaped specimens were cut for tear testing in accordance with the ISO 37:2005 standards. Specimens were placed in the pneumatic grips of the tensiometer and specimens analysed at a loading rate of $100 \mathrm{~mm} / \mathrm{min}$ and $500 \mathrm{~mm} / \mathrm{min}$ for tensile and tear testing respectively. The data was captured using Bluehill software. Ultimate tensile strength, strain at break, Young's modulus and tear strength values were recorded. Unpaired t-testing using GraphPad Prism software Version 6 was used to analyse differences between irradiated and non-irradiated samples. 


\section{ATR-FTIR of Implant Shells}

Fourier Transform Infrared Spectra (FTIR) recordings were obtained to determine the surface chemical fingerprint of the implants using a Jasco FT/IR 4200 Spectrometer with a diamond attenuated total reflectance accessory (Diamond Miracle ATR, Pike Technologies, US). A total of 5 shell samples from each of the radiated groups and the control group were analysed. From an average of 30 scans a spectra was produced over a range of $600 \mathrm{~cm}^{-1}$ to $4000 \mathrm{~cm}^{-1}$ with a resolution of $4 \mathrm{~cm}^{-1}(\mathrm{n}=5)$ for each group. The resulting spectra and the peak spectral intensities were identified and one-way ANOVA was performed using Graph Pad Prism software Version 6 to detect changes between the groups.

\section{Contact Angle Measurements of Implant Shells}

Using a DSA 100 Krüss Goniometer, wettability analysis was performed on the implant samples from each of the 3 groups. Using the sessile drop technique, $5 \mathrm{ul}$ of deionized water was dropped onto the samples using an automated syringe with 10 seconds of dispensing and analysis was performed using the Drop Analysis software (EasyDrop DSA200, KrüSS) at room temperature. Four samples from specimens from each group were tested three times $(n=12)$ and statistical analysis performed using Graph Pad Prism software Version 6.

\section{Cell Metabolism and Morphology}

In order to assess cell metabolic activity and number, human dermal fibroblasts (HDFa) were cultured in Dulbecco's modified eagle medium supplemented with $10 \%$ foetal bovine serum and 1\% strep/ben pen. HDFa cells used were between passage 7 and 11. From each of the three groups: silicone shell radiated at $40.05 \mathrm{~Gy}$, silicone shell radiated at $2.67 \mathrm{~Gy}$ and non-irradiated (control) silicone shell, $6 \mathrm{~mm}$ round disc cut samples $(\mathrm{n}=8)$ from were sterilized by being placed in $1 \%$ Triton $\mathrm{X}$ for 1 hour, washed twice in PBS followed by $70 \%$ ethanol followed by washing twice in PBS. The discs $(n=8)$ were placed in a 96 well plate, covered with $100 \mu 1$ of warmed DMEM for approximately 2 hours then seeded with HDFa cells at a density of $5 \times 10^{4}$ cells $/ \mathrm{cm}^{2}$. Cells seeded onto tissue culture plastic served as a positive control and media only wells provided a negative control. Cells were incubated at $37^{\circ} \mathrm{C}$ at $5 \%$ $\mathrm{CO} 2$ in air. 


\section{Alamar Blue ${ }^{\mathrm{TM}}$ assay}

Cell metabolism was assessed using Alamar Blue ${ }^{\mathrm{TM}}$ assay (Invitrogen, Paisley, UK) and conducted in accordance with protocol guidelines on days 1, 3 and 7. Fluorescence was measured using a Fluoroskan Ascent ${ }^{\mathrm{TM}}$ Fluorescence Plate Reader (ThermoScientific, USA) at excitation and emission wavelength of 530nm and 620nm $(\mathrm{n}=8)$. Two way ANOVA analysis was performed using GraphPad Prism version 6 using Tukey's correction

\section{Cell Morphology}

Using commercially available fluorescent green cytoplasmic actin (Alexa Fluor 488 Phalloidin, Molecular Probes, ThermoScientific ${ }^{\mathrm{TM}}$, UK) and fluorescent blue nuclei staining kit (Vectashield Antifade Mounting Medium with DAPI, Vector Laboratories, USA), cell morphology was examined at day 7 of seeded HDFa cells on tissue culture plastic and on non-irradiated silicone implant shells. This was performed in accordance to the manufacturers protocols. Images were captured using an EVOS Fluorescent Microscope (EVOS FL Imaging System, ThermoScientific ${ }^{\mathrm{TM}}$, UK).

\section{Statistical Analysis}

All statistics were performed using GraphPad Prism software version 6. 


\section{Results}

\section{Material Mechanical Properties}

There were no significant differences in maximal tensile strength (Fig. 1A) and tear strength (Fig. 1C) between each of the groups using unpaired t-testing. There was no significant difference in Young's modulus between the control and full treatment dose group $(40.05 \mathrm{G})$ but there was a significant decrease in the 2.67 Gy group (Fig. 1D). A significant reduction in the maximum elongation strain at break was, however, evident (Fig. 1B, $\mathrm{p}=0.001 \leqslant 0.05$ ) suggesting the samples are less flexible under strain following irradiation.

\section{Material Surface Chemistry}

\section{ATR-FTIR}

Analysis of the material surface properties was performed using ATR-FTIR. Despite some spectral difference observed upon spectral subtraction (Fig. 2A) of the ATRFTIR spectra, there was no significance difference between the peak heights at $784 \mathrm{~cm}^{-1}$ corresponding to $-\mathrm{CH}_{3}$ rocking and $-\mathrm{Si}-\mathrm{C}$-stretching in $-\mathrm{Si}-\mathrm{CH}_{3}(\mathrm{p}=0.33)$ one way ANOVA, parametric data), at $1004 \mathrm{~cm}^{-1}$ corresponding to the asymmetric stretching of -Si-O-Si- $(\mathrm{p}=0.87)$, one -way ANOVA, parametric data) and at $1257 \mathrm{~cm}^{-1}$ corresponding to symmetric bending of $-\mathrm{CH}_{3}$ in $-\mathrm{Si}-\mathrm{CH}_{3}(\mathrm{p}=0.67$, one way ANOVA parametric data) as shown in Fig. 3. These peak heights measurements were $n=15$ ) were analysed independently of each other. 


\section{Contact angle measurements (wettability)}

On analysis of the contact angle measurements there was no significant differences detected between each of the groups ( $\mathrm{p}=0.23$, Kruskal Wallis test) as shown in Fig. 4.

\section{Cell Metabolism and Morphology}

\section{Cell metabolism}

Cell metabolic activity increased with time (Day 1 and Day 7) as cells proliferated on all samples, but no significant difference was detected between irradiated ( $\mathrm{n}=12)$ (40.05Gy) and non-irradiated silicone $(\mathrm{n}=12) \quad(\mathrm{p}=0.79$, 2-way ANOVA) (Fig. 5). Significant difference was detected in cell activity between the days $(p<0.0001)$.

\section{Cell Morphology (Immunofluorescence staining)}

The cell morphology of cells grown on tissue culture plastic (TCP) demonstrated highly aligned, thin, long, spindle like projections in parallel with an abundance of nuclei. In contrast, the cells cultured upon non-irradiated silicone breast implant shells showed thickened projections with random orientation with fewer nuclei evident 


\section{Discussion}

As the indications for PMRT are expanding [6] and as patients undergoing implant based breast reconstruction who receive PMRT have been reported to have a increased rate of surgical complications such as capsular contracture and revisional surgery [13] there is a clinical need to understand the mechanism of failure, which could lead to the creation of new materials or different treatment approaches. This is further supported by a recent systematic review by Lam et al. who reported patients undergoing a two stage implant based breast reconstruction demonstrated a higher rate of complications (capsular contracture, reconstructive failure and poor cosmetic outcome) of $18.6 \%$ in those receiving PMRT versus $3.1 \%$ ( $\mathrm{p}<0.00001)$ in those without [19]. This study sought to establish if the physicochemical properties and consequently cellular response to silicone breast implants change following radiation treatment.

This study showed there were no significant changes in tensile strength between the groups in keeping with previous literature [18] and tear strength (Fig.1). Young's modulus was not significant changed following full dose radiation (40.05Gy) but showed a decrease following single treatment fraction dose (2.67 Gy). Maximal elongation at break was significantly reduced after both single treatment fraction radiation dose $(2.67 \mathrm{~Gy})$ and after full dose radiation (40.05 Gy) suggesting that 
samples are less flexible after irradiation. Limitations to this study include testing implants of one type and from one manufacturer (Mentor Siltex ${ }^{\mathrm{TM}}$ Contour Profile ${ }^{\mathrm{TM}}$ Becker TM 35 Expander implants). These implants were available and commonly used for breast reconstruction in the unit but the limited amount of sample material available for analysis may have influenced the results. Furthermore, our methods of material characterization assessed bulk mechanical properties in dry conditions at room temperature that may not be consistent with in-vivo conditions. There may have been micro-mechanical changes that were therefore not detected and further modalities of investigations including atomic force microscopy are warranted.

Contact angle measurements, performed to assess the hydrophobicity of the tested material that directly influences protein and cell attachment, were not significantly different between the irradiated and non-irradiated groups. ATR-FTIR analysis showed no significant differences detected between the spectral peaks amongst the three groups. This is not in keeping with Ribuffo et al. [18] who detected changes in ATR-FTIR analysis following treatment dose radiation therapy with the observance of smaller fragments suggesting scission of the polymers chains and degradation of the shells [18]. Although sample sizes were similar, this may possibly be explained by the higher radiation dose delivered in the study (50 Gy versus $40.05 \mathrm{~Gy}$ ) delivered over a total of 25 fractions over 5 weeks in comparison to this study which delivered the full treatment radiation dose in one sitting.

Cell metabolism of HDFa cells seeded on shells from each of the groups revealed no significant differences as demonstrated by Alamar Blue assay. Immunofluorescence staining revealed the effect on cell orientation and morphology between the 'smooth' TCP surface and the textured surface of the silicone implant. The cells grown on the TCP showed highly aligned, stretched out fibroblasts with increased nuclei in comparison to the cells grown upon the textured non-irradiated silicone implant which showed random orientation of shortened cells and reduced numbers of cells. This is in-keeping with clinical studies describing increased rates of capsular contracture in patients with smooth implants in comparison to textured implants [20].

Several theories as to the mechanism of capsular contracture exists including biofilm formation leading to chronic inflammation [21], surgical handling of the implant, 
peri-operative complications including haematoma and seroma, implant filler, radiation therapy and submuscular implant placement [10] but the actual mechanism is not fully understood and thought to be multi-factorial. It is well documentated that radiation causes damage to normal cells through damage to DNA and cellular components leading to alterations in the cells signaling pathways could explain the increased rate of implant failure in those patients receiving PMRT [22]. Another possible factor could be the tumour micro-environment which could be contributing to implant failure as those patients receiving PMRT may in general have more advanced disease than patients who do not require PMRT.

This study shows that treatment dose radiation therapy administered to the silicone breast implant does not have an overall effect on the mechanical, surface chemistry and fibroblast cellular response and this is in keeping with previous literature examining the effect of radiation on PDMS based materials $[16,17]$. However, of note these studies used significantly greater doses of radiation for the purpose of examining the effect of material sterilisation than the doses required in the clinical setting and thus used in our experiment. Further in-vitro research is required to examine the effect of radiation therapy to the cells and the silicone breast implants in parallel to elucidate the mechanisms of development of capsular contracture and breast implant failure. 



\section{References}

[1] Chang JM, Kosiorek HE, Dueck AC, Casey WJ, Rebecca AM, Mahabir R, et al. Trends in mastectomy and reconstruction for breast cancer; a twelve year experience from a tertiary care center. Am J Surg 2016;212:1201-10. doi:10.1016/j.amjsurg.2016.08.020.

[2] Kummerow KL, Du L, Penson DF, Shyr Y, Hooks MA. Nationwide Trends in Mastectomy for Early-Stage Breast Cancer. JAMA Surg 2015;150:9. doi:10.1001/jamasurg.2014.2895.

[3] Al-Ghazal SK, Fallowfield L, Blamey RW. Comparison of psychological aspects and patient satisfaction following breast conserving surgery, simple mastectomy and breast reconstruction. Eur J Cancer 2000;36:1938-43.

[4] Ragaz J, Jackson SM, Le N, Plenderleith IH, Spinelli JJ, Basco VE, et al. Adjuvant radiotherapy and chemotherapy in node-positive premenopausal women with breast cancer. N Engl J Med 1997;337:956-62. doi:10.1056/NEJM199710023371402.

[5] Overgaard M, Hansen PS, Overgaard J, Rose C, Andersson M, Bach F, et al. Postoperative radiotherapy in high-risk premenopausal women with breast cancer who receive adjuvant chemotherapy. Danish Breast Cancer Cooperative Group 82b Trial. N Engl J Med 1997;337:949-55.

doi:10.1056/NEJM199710023371401.

[6] Recht A, Comen EA, Fine RE, Fleming GF, Hardenbergh PH, Ho AY, et al. Postmastectomy Radiotherapy: An American Society of Clinical Oncology, American Society for Radiation Oncology, and Society of Surgical Oncology Focused Guideline Update. J Clin Oncol 2016;34:4431-42. doi:10.1200/JCO.2016.69.1188. 
[7] Spear SL, Murphy DK, Allergan Silicone Breast Implant U.S. Core Clinical Study Group. Natrelle round silicone breast implants: Core Study results at 10 years. Plast Reconstr Surg 2014;133:1354-61.

doi:10.1097/PRS.0000000000000021.

[8] Colaris MJL, de Boer M, van der Hulst RR, Cohen Tervaert JW. Two hundreds cases of ASIA syndrome following silicone implants: a comparative study of 30 years and a review of current literature. Immunol Res 2017;65:120-8. doi:10.1007/s12026-016-8821-y.

[9] Adams WP. Capsular Contracture: What is It? What Causes It? How Can It Be Prevented and Managed? Clin Plast Surg 2009;36:119-26. doi:10.1016/j.cps.2008.08.007.

[10] Berry MG, Cucchiara V, Davies DM. Breast augmentation: Part II - adverse capsular contracture. J Plast Reconstr Aesthetic Surg 2010;63:2098-107. doi:10.1016/j.bjps.2010.04.011.

[11] Anker CJ, Hymas R V, Ahluwalia R, Kokeny KE, Avizonis V, Boucher KM, et al. The Effect of Radiation on Complication Rates and Patient Satisfaction in Breast Reconstruction using Temporary Tissue Expanders and Permanent Implants. Breast J 2015;21:233-40. doi:10.1111/tbj.12395.

[12] Cordeiro PG, Albornoz CR, McCormick B, Hu Q, Van Zee K. The Impact of Postmastectomy Radiotherapy on Two-Stage Implant Breast Reconstruction. Plast Reconstr Surg 2014;134:588-95. doi:10.1097/PRS.0000000000000523.

[13] Magill LJ, Robertson FP, Jell G, Mosahebi A, Keshtgar M. Determining the outcomes of post-mastectomy radiation therapy delivered to the definitive implant in patients undergoing one- and two-stage implant-based breast reconstruction: A systematic review and meta-analysis. J Plast Reconstr 
Aesthet Surg 2017;70:1329-35. doi:10.1016/j.bjps.2017.05.057.

[14] Nava MB, Pennati AE, Lozza L, Spano A, Zambetti M, Catanuto G. Outcome of different timings of radiotherapy in implant-based breast reconstructions. Plast Reconstr Surg 2011;128:353-9. doi:10.1097/PRS.0b013e31821e6c10.

[15] Vandeweyer E, Deraemaecker R. Radiation therapy after immediate breast reconstruction with implants. Plast Reconstr Surg 2000;106:56-8; discussion 59-60.

[16] Briganti E, Kayal T Al, Kull S, Losi P, Spiller D, Tonlorenzi S, et al. The effect of gamma irradiation on physical-mechanical properties and cytotoxicity of polyurethane-polydimethylsiloxane microfibrillar vascular grafts. J Mater Sci Mater Med 2010;21:1311-9. doi:10.1007/s10856-009-3943-6.

[17] Simmons A, Hyvarinen J, Poole-Warren L. The effect of sterilisation on a poly(dimethylsiloxane)/poly(hexamethylene oxide) mixed macrodiol-based polyurethane elastomer. Biomaterials 2006;27:4484-97. doi:10.1016/j.biomaterials.2006.04.017.

[18] Ribuffo D, Torto F Lo, Giannitelli SM, Urbini M, Tortora L, Mozetic P, et al. The effect of post-mastectomy radiation therapy on breast implants: Unveiling biomaterial alterations with potential implications on capsular contracture 2015. doi:10.1016/j.msec.2015.07.015.

[19] Lam TC, Hsieh F, Boyages J. The Effects of Postmastectomy Adjuvant Radiotherapy on Immediate Two-Stage Prosthetic Breast Reconstruction. Plast Reconstr Surg 2013;132:511-8. doi:10.1097/PRS.0b013e31829acc41.

[20] Wong C-H, Samuel M, Tan B-K, Song C. Capsular contracture in subglandular breast augmentation with textured versus smooth breast implants: a systematic review. Plast Reconstr Surg 2006;118:1224-36. 
doi:10.1097/01.prs.0000237013.50283.d2.

[21] Rieger UM, Mesina J, Kalbermatten DF, Haug M, Frey HP, Pico R, et al. Bacterial biofilms and capsular contracture in patients with breast implants. $\mathrm{Br}$ J Surg 2013;100:768-74. doi:10.1002/bjs.9084.

[22] Stone HB, Coleman CN, Anscher MS, McBride WH. Effects of radiation on normal tissue: Consequences and mechanisms. Lancet Oncol 2003;4:529-36. doi:10.1016/S1470-2045(03)01191-4. 

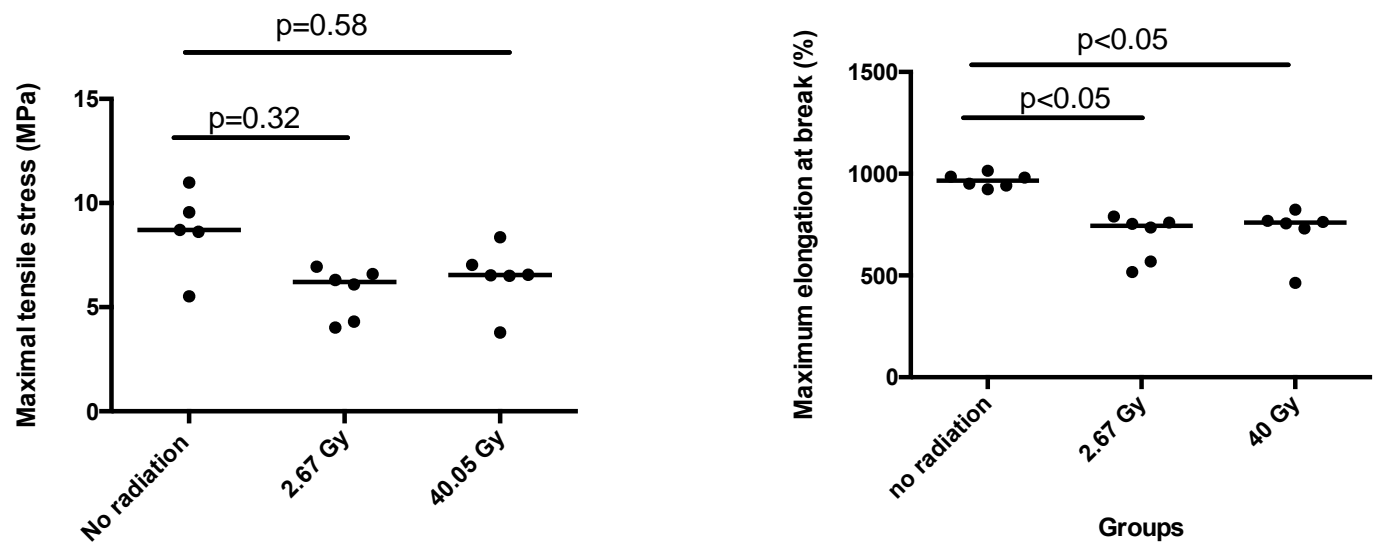

C Maximum Tear Strength (N/mm)

D Young's modulus (MPa)
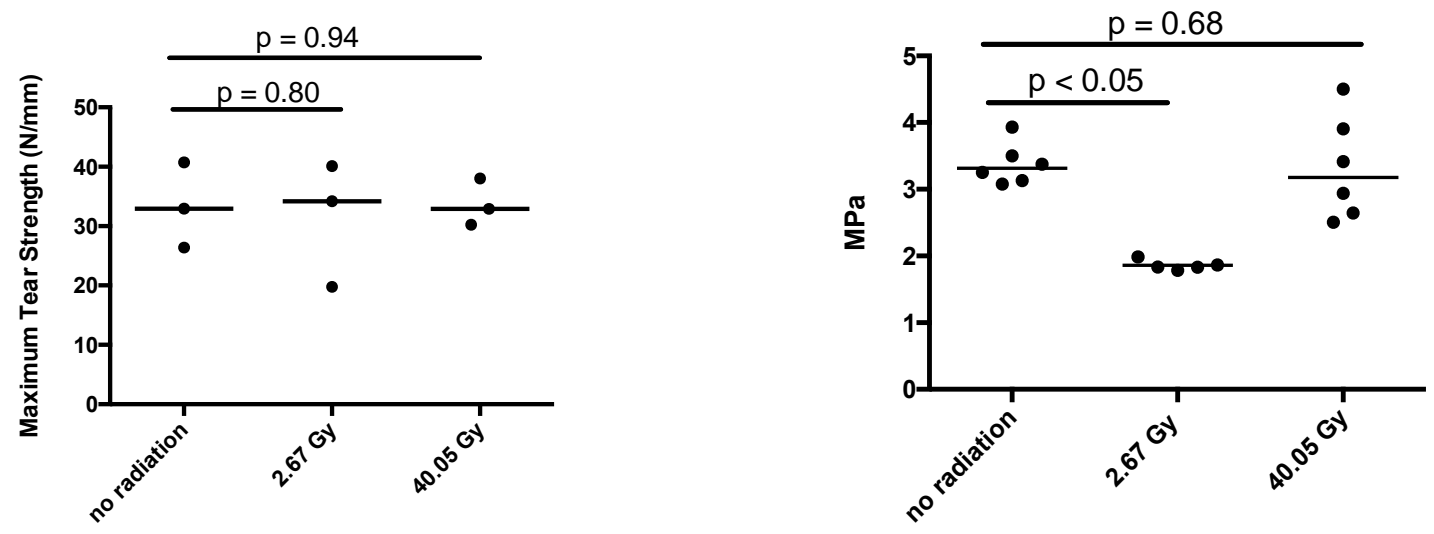

Figure 1: Mechanical Characterisation of Samples

No significant difference detected in A. maximal tensile strength and C. maximal tear strength. Significant differences were seen in C. maximum elongation at break. No significant difference in Young's modulus detected between control and full dose radiation groups $\mathbf{D}$. 


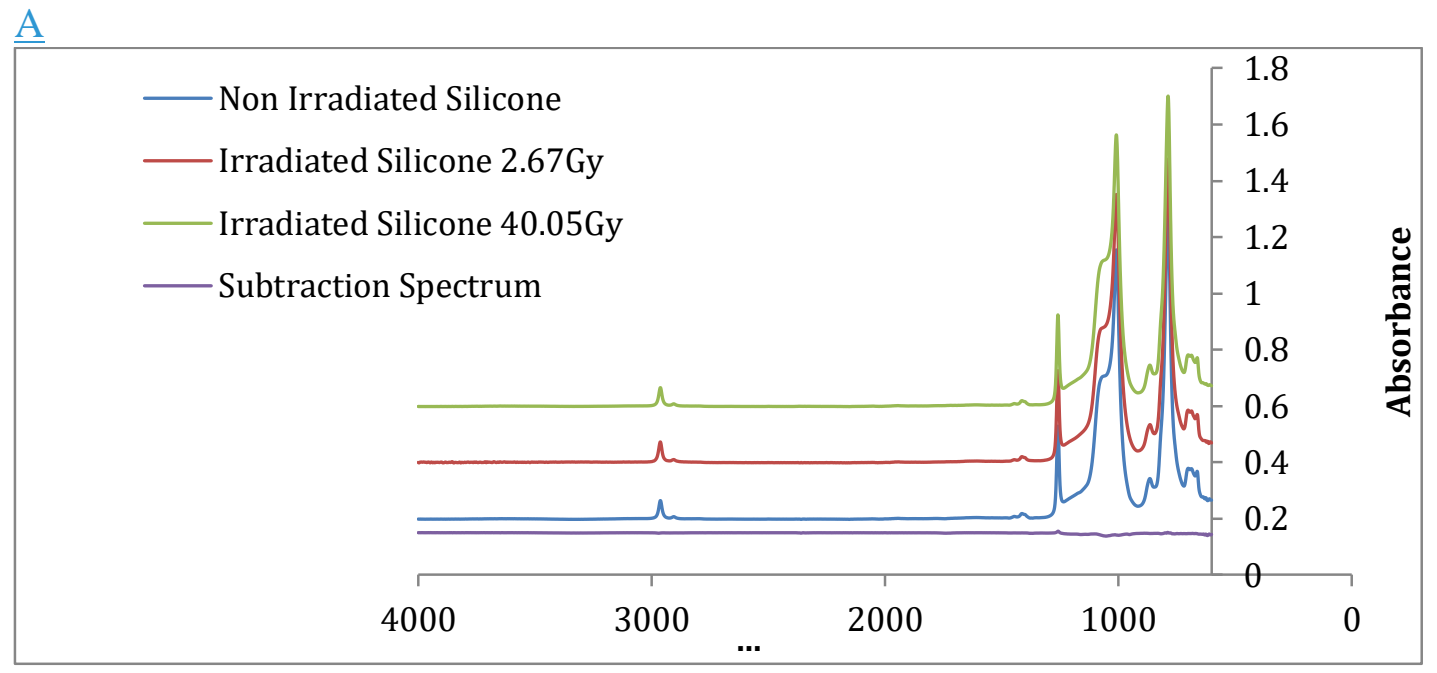

$\underline{\mathbf{B}}$

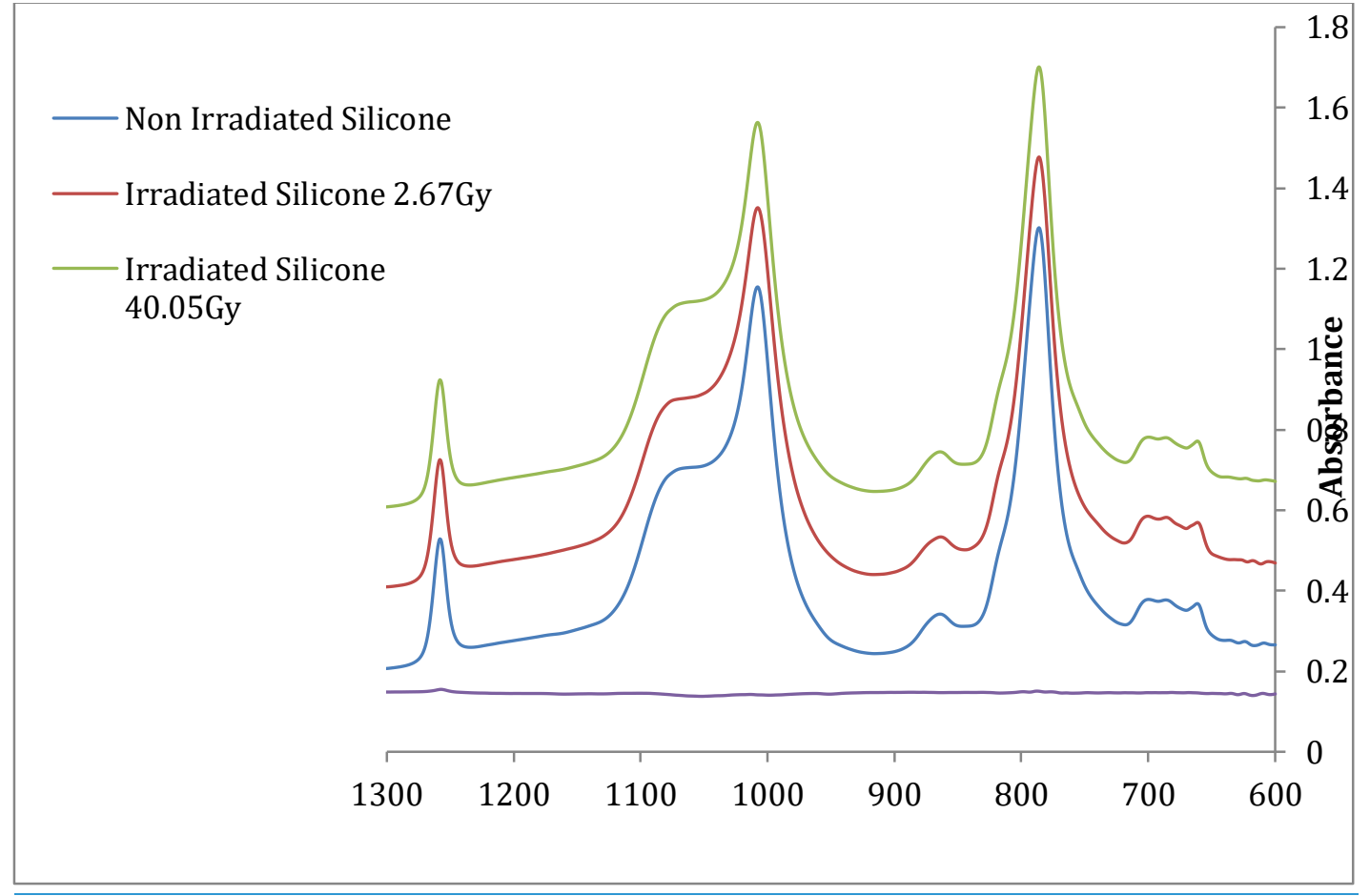

Figure 2: A. Overlaid ATR-FTIR spectra of non-irradiated (control) and irradiated silicone breast implant shells at $2.67 \mathrm{~Gy}$ and at $40.05 \mathrm{~Gy}$ offset by +0.2 with subtraction spectrum (Non Irradiated Silicone - Irradiated Silicone 40.05 Gy). B. Spectra between 600 and $1300 \mathrm{~cm}^{-1}$ wavelength showing substraction spectrum. 


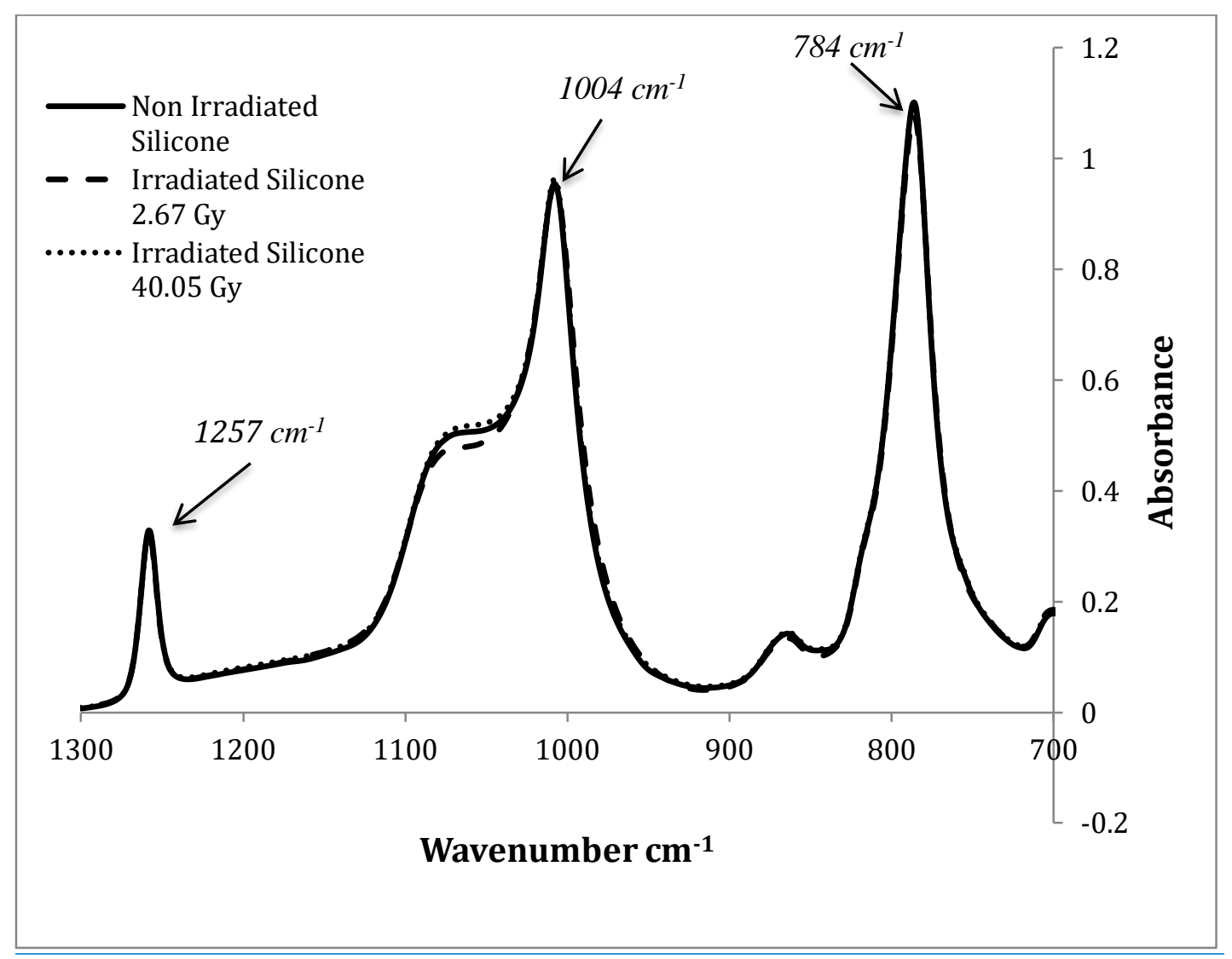

Figure 3: ATR-FTIR spectra from wavenumber $700-1300 \mathrm{~cm}^{-1}$ showing overlaid spectra from non irradiated and irradiated specimens. No significant differences in spectra height seen at peak $784 \mathrm{~cm}^{-1}, 1004 \mathrm{~cm}^{-1}$ and $1257 \mathrm{~cm}^{-1}$ 


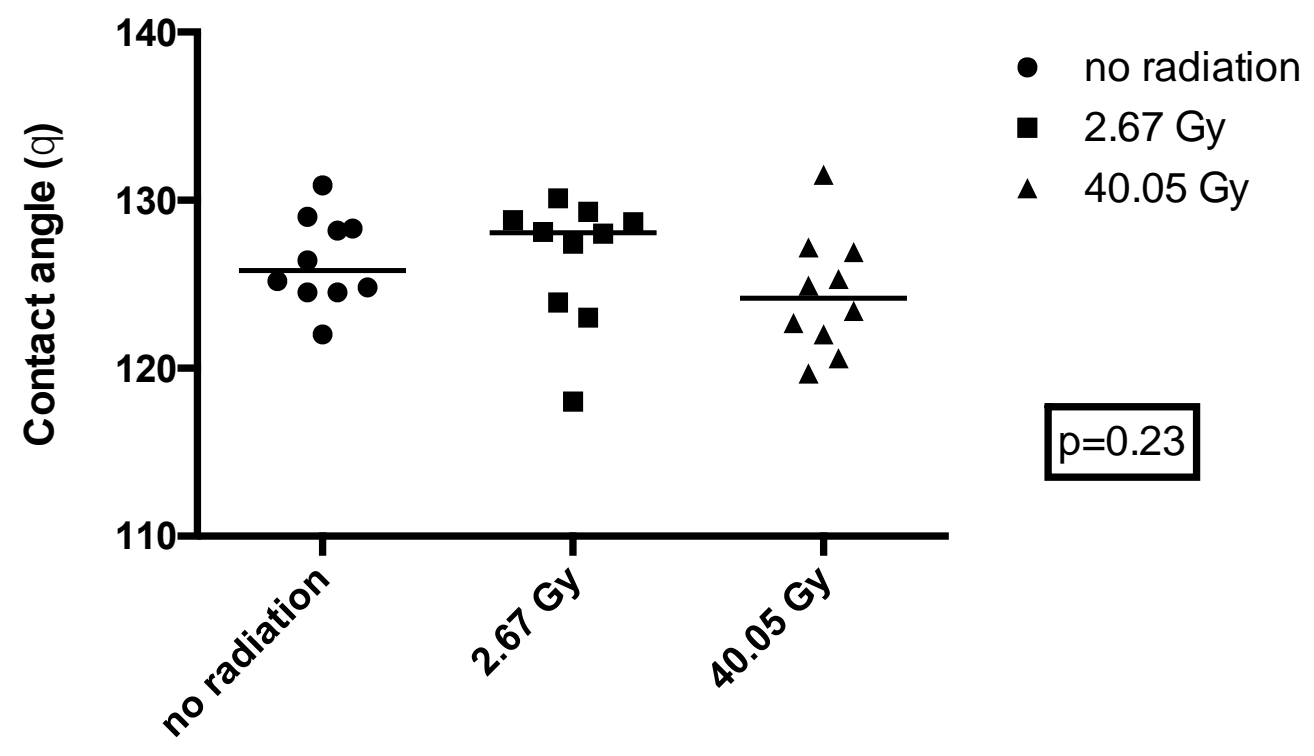

Figure 4: Contact Measurements showed no significant differences in each of the 3 groups ( $\mathrm{p}=0.23)$, Kruskal-Wallis test) 


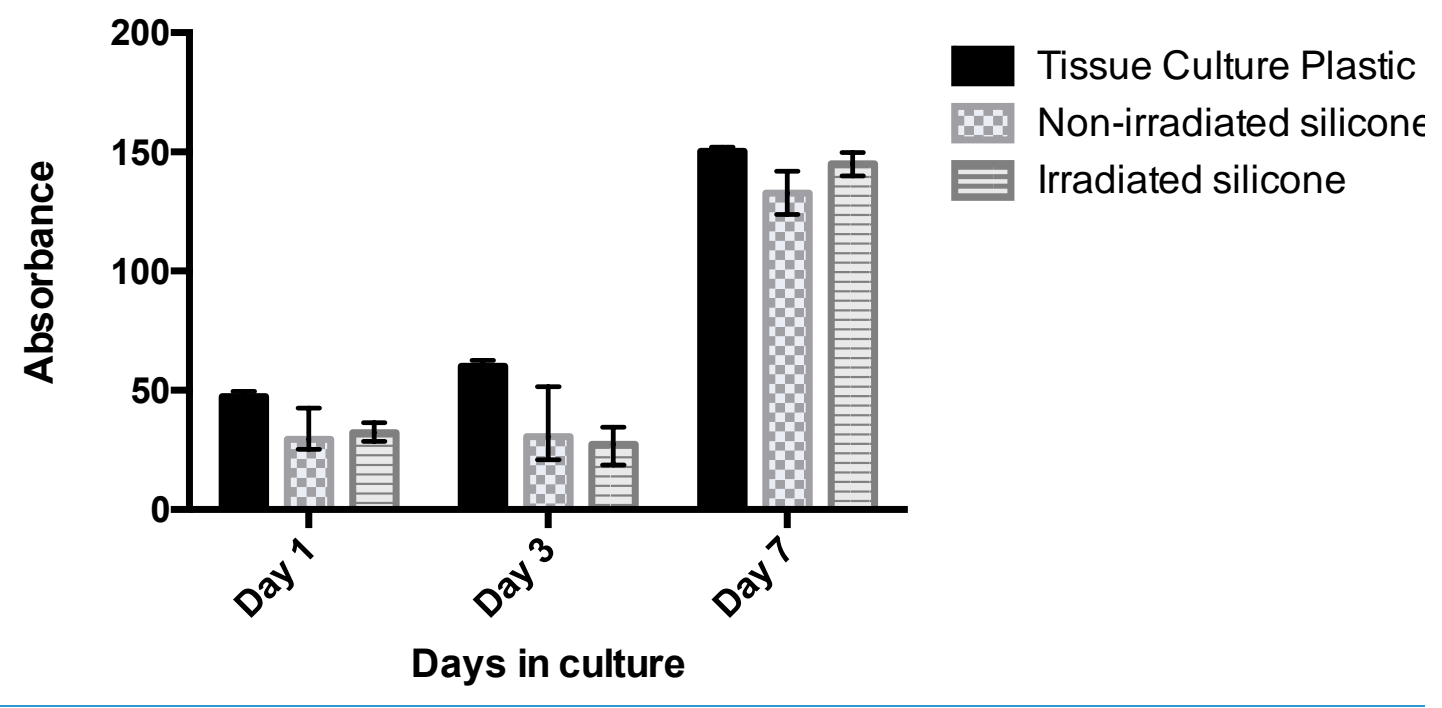

Figure 5: Cell metabolism on tissue culture plastic (TCP), cells grown on non-

irradiated silicone breast implant shell and silicone breast implant shell subjected to full treatment dose radiation (40.05 Gy) (Irradiated Silicone) as assessed by Alamar Blue ${ }^{\mathrm{TM}}$ assay over 7 days. No significant differences were detected between the tested material groups ( $p=0.79$, using 2-way ANOVA test). 


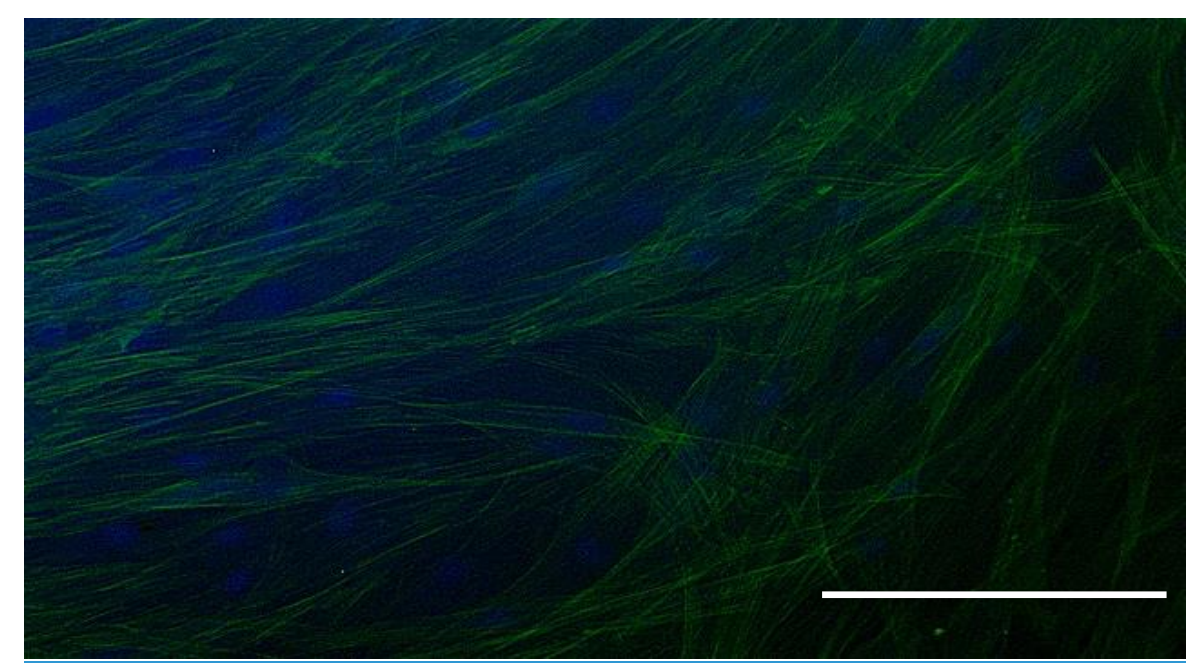

Fig. 6A. Cells seeded on TCP at Day 7

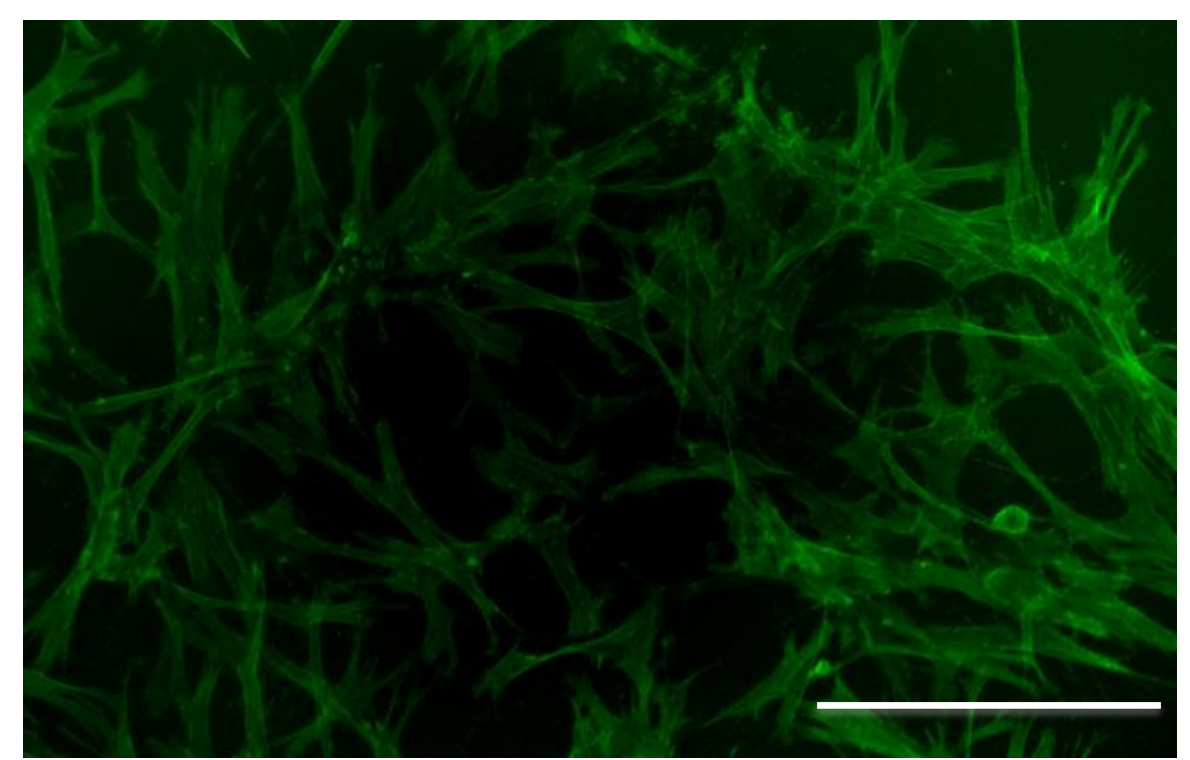

Fig. 6B. Cells seeded on non-irradiated Silicone Breast implant shells at Day 7

Figure 6: Fluorescent images capturing HDFa cells cultured at Day 7 stained with fluorescent F-actin (green) and DAPI nuclei (blue) staining on A. TCP versus cells seeded on B. non-irradiated Silicone Breast implant shells. The white line represents $\underline{200 \mu \mathrm{m}}$. 